\title{
The impact of Allergan implant-related issues on patients with breast implants for reconstruction
}

\author{
Ji Min Kim', Woo Jin Song', \\ Hyun Gyo Jeong ${ }^{2}$, Sang Gue Kang ${ }^{1}$ \\ ${ }^{1}$ Department of Plastic and \\ Reconstructive Surgery, Soonchunhyang \\ University Seoul Hospital, Seoul; \\ ${ }^{2}$ Department of Plastic and \\ Reconstructive Surgery, Soonchunhyang \\ University Gumi Hospital, Gumi, Korea
}

This article was presented at the 10th Research \& Reconstruction Forum on July 24, 2020.

\begin{abstract}
Background In August 2019, the Ministry of Food and Drug Safety and the Korean Society of Plastic and Reconstructive Surgeons confirmed the first case report of breast implant-associated anaplastic large cell lymphoma (BIA-ALCL) in Korea and provided recommendations. This study was conducted to evaluate the impact of the first case report of BIA-ALCL on patients and whether the impact could be mitigated through expert recommendations.

Methods A retrospective chart review was performed of patients who underwent implant-based breast reconstruction using Biocell textured breast implants at Soonchunhyang University Hospital. After the first case report of BIA-ALCL and informing the patients via text messages, the pattern of outpatient visits and the proportion and indications of outpatient office visitors who received reoperations were analyzed.

Results After the first BIA-ALCL case report in Korea, 12 patients underwent reoperations due to other complications identified incidentally, while only three patients underwent prophylactic surgery. Among the patients who underwent reoperation after the Allergan issues, $76.7 \%$ received implant exchange. There was no significant difference in the proportion of reoperation types before and after the Allergan issues and the transmission of text messages $(P=0.700)$.

Conclusions Despite the major issues, outpatients were managed successfully according to our recommendations. The study found a low rate of regular outpatient visits; however, our efforts increased this rate. Therefore, methods for appropriate information delivery are needed for outpatient visits, as well as a system for regular monitoring. As experts, plastic surgeons should provide responsible leadership in this situation to relieve patients' concerns.
\end{abstract}

Keywords Breast reconstruction / Breast implant / Complications / Lymphoma, large-cell, anaplastic / Patient compliance

\section{INTRODUCTION}

Breast implant-associated anaplastic large cell lymphoma (BIAALCL) is a rare type of non-Hodgkin's T cell lymphoma that oc-

Received: Jun 16, 2021 Revised: Jun 29, 2021 Accepted: Aug 17, 2021 Correspondence: Sang Gue Kang Department of Plastic and Reconstructive Surgery, Soonchunhyang University Seoul Hospital, 59 Daesagwan-ro, Yongsan-gu, Seoul 04401, Korea

Tel: +82-2-709-9283, Fax: +82-2-705-3687, E-mail: sgkang@schmc.ac.kr

Copyright @ 2021 The Korean Society for Aesthetic Plastic Surgery.

This is an Open Access article distributed under the terms of the Creative Commons At tribution Non-Commercial License (https://creativecommons.org/licenses/by-nc/4.0/) which permits unrestricted non-commercial use, distribution, and reproduction in any medium, provided the original work is properly cited. $\quad w w w . e-a a p s . o r g$ curs around implants [1]. BIA-ALCL is especially related to textured types of implants, and its incidence rate is known to increase with the degree of texture $[2,3]$. The clinical features of BIA-ALCL are large fluid collection, delayed seroma, or a capsular mass $[1,4]$. The lifetime prevalence of BIA-ALCL was estimated at about 1 in 30,000 patients and the median time to diagnosis in patients exposed to textured implants was about 10 years $[5,6]$.

Since 2011, the United States Food and Drug Administration (U.S. FDA) has consistently warned of the risks of BIA-ALCL and recommended a recall of the Biocell textured breast implant (Allergan, Inc.) and tissue expander in July 2019 [7]. The recall followed similar actions worldwide, including in France, Canada, and Australia, where the use of textured implants is much higher [8]. Korea has the third-highest number of cosmetic procedures performed 
every year, after the U.S. and Brazil, and breast implant surgery has also increased significantly [3]. According to a report of the Korean Ministry of Food and Drug Safety (KMFDS), more than 100,000 Biocell textured breast implants were distributed in Korea from 2007 to 2018 [9].

In August 2019, the first BIA-ALCL case was reported in Korea, and BIA-ALCL became an issue through the media [10]. Patients who underwent breast surgery with implants felt substantial anxiety about this issue; in particular, patients who underwent reconstruction after mastectomy were very worried that their risk of cancer could increase again. Meanwhile, some hospitals promoted the replacement of Biocell textured breast implants and removed the capsules. In response to this, the Korean Society of Plastic and Re-

\section{[Hospital]}

As 'Anaplastic Large Cell Lymphoma' has recently occurred in patients with textured breast implants from Allergan, we will inform patients of safety information according to the guidelines of the Ministry of Food and Drug Safety.

- If there are no symptoms, preventive removal is not recommended (including the rate of occurrence and risk of preventive removal)

- If you have suspicious symptoms such as a change in breast size, touching a hard lump, ulcers or redness on the skin, visit a medical institution for examination

- Recommended regular checkup once a year after 1 year of surgery

Fig. 1. The text message for patients with textured breast implants. constructive Surgeons (KSPRS) and the Korean Food and Drug Administration (i.e., the KMFDS) proposed some recommendations.

The recommendations were as follows: (1) considering the low incidence of BIA-ALCL, the risks of anesthesia, and postoperative complications after surgery, the prophylactic removal of breast implants in asymptomatic patients is not recommended; (2) for symptoms suspicious of BIA-ALCL, patients should promptly visit a medical institution with a breast specialist for an examination; or (3) even if there are no abnormal symptoms, regular checkups are necessary once a year.

In accordance with these recommendations, our institution also sent cellphone text messages in October 2020 to patients who had received Biocell textured breast implants, and counseling was conducted through outpatient visits and telephone calls (Fig. 1).

This was the first time that such large-scale breast implant-related issues occurred in Korea. In this study, we investigated how the Allergan issues in Korea affected patients and whether their concerns could be managed through expert recommendations. Therefore, we indirectly investigated patients' responses after the Allergan issues and information delivery by quantifying the rate of reoperation and the rate of replacement or removal of implants during reoperation.

\section{METHODS}

\section{Study design}

A single-center, retrospective chart review was performed, focusing on patients' responses after the first BIA-ALCL case report in

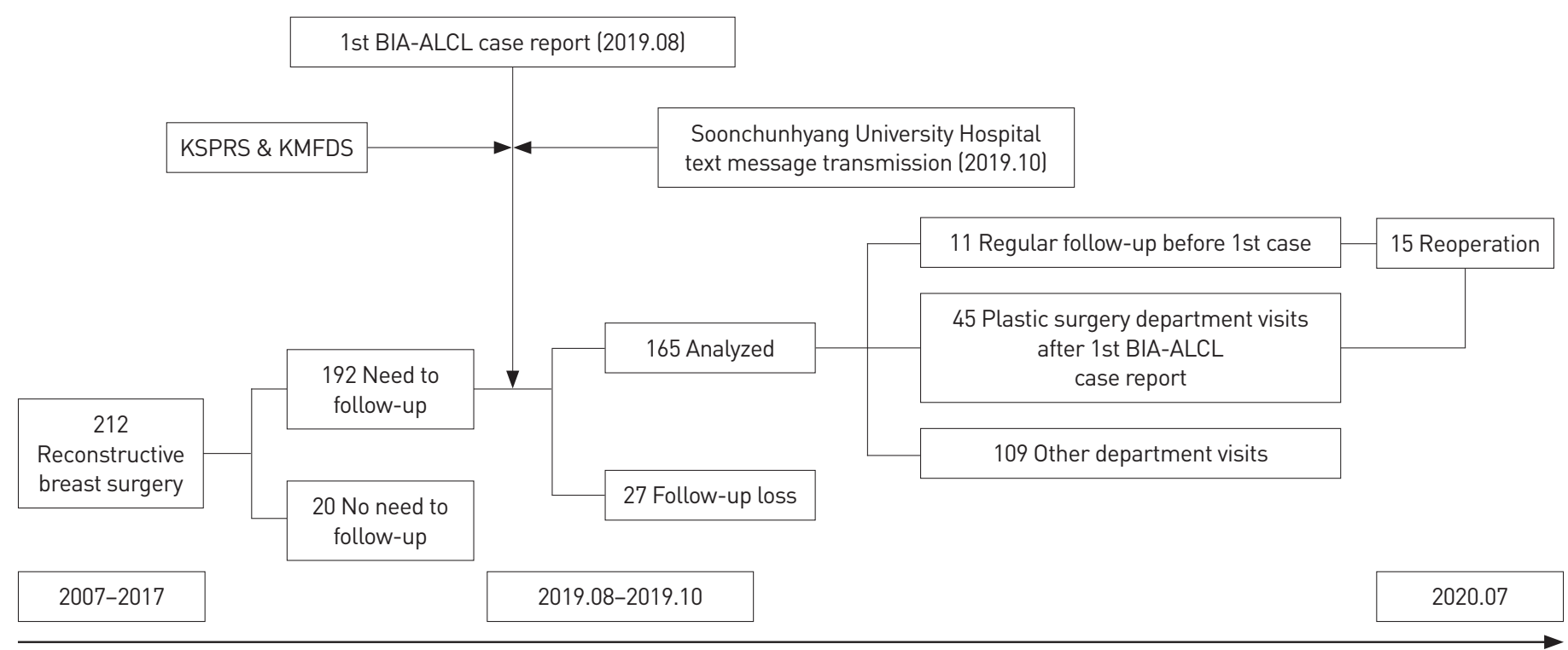

Fig. 2. Flow diagram of the study of patient responses after the first BIA-ALCL case in Korea. BIA-ALCL, breast implant-associated anaplastic large cell lymphoma; KSPRS, Korean Society of Plastic and Reconstructive Surgeons; KMFDS, Korean Ministry of Food and Drug Safety. 
Korea. The study was approved by the Institutional Review Board of Soonchunhyang University Hospital (IRB No. 2021-01-011) and was performed in accordance with the principles of the Declaration of Helsinki.

\section{Inclusion and exclusion criteria}

The Inclusion criteria were as follows: (1) from January 2011 to December 2017, patients who underwent implant-based breast surgery with Biocell textured breast implants at the Soonchunhyang University Hospital and (2) patients who underwent surgery for reconstruction purposes. The exclusion criteria were as follows: (1) patients who underwent implant-based breast surgery for cosmetic purposes; (2) patients with no need for follow-up: patients who died, underwent reoperation for removal of an implant, or had the implant replaced with a different type of implant before the first case report of BIA-ALCL; or (3) patients lost to follow-up: no visits to our institution, including other departments, for the last 2 years.

A flow diagram of this study of responses after the first BIA-ALCL case in Korea is shown in Fig. 2. From 2007 to 2017, patients who underwent breast reconstructive surgery using Biocell textured breast implants at Soonchunhyang University Seoul Hospital were identified by searching the product codes, and cellphone text messages were sent in October 2019.

After delivering the recommendations, the patients who requested telephone counseling were advised to visit the outpatient clinic. In the outpatient clinic, a physical examination was performed, and patients with suspicious findings underwent additional imaging studies. Reoperation was performed upon the patient's request due to concerns about the risk of BIA-ALCL, even though we explained the recommendations, or when other complications requiring reoperation were found incidentally. In case of reoperation, a recommendation was made to replace the textured implant with a smooth implant rather than removing it without replacement, and the decision of whether to perform capsulectomy was made according to the findings in the operating room.

A retrospective analysis was conducted approximately 1 year after the first domestic BIA-ALCL case report. Information on prior operation records, outpatient office records, and reoperation records was collected. The analysis explored the pattern of outpatient visits and the proportion and indications of outpatient office visitors who underwent reoperations after receiving text messages following the first case report of BIA-ALCL.

To evaluate the impact of the first BIA-ALCL case report and compliance with the recommendations, 20 patients who underwent breast reconstructive surgery using Biocell textured breast implants at Soonchunhyang University Seoul Hospital and were reoperated before the first BIA-ALCL case report were selected as a control group. The proportion of types and indications of reoperation were compared between before and after the first BIA-ALCL case report.

\section{Statistical analysis}

Statistical analysis was conducted using SPSS version 23.0 (IBM Corp., Armonk, NY, USA). The Fisher exact test was employed for the statistical analyses regarding the difference in the timing of reoperation based on the first case report of BIA-ALCL and reoperation types, and differences exhibiting a P-value $<0.05$ were considered statistically significant.

\section{RESULTS}

Of the 240 patients who underwent surgery using Biocell textured breast implants at our institution, the majority $(212,88.3 \%)$ did so for reconstruction purposes. Among the patients who received breast implants for reconstruction, 20 patients who did not need follow-up were excluded due to death or replacement with other types of implants or removal of the Biocell textured breast implant for complications such as capsular contracture, infection, breast cancer recurrence, or implant rupture. Furthermore, 27 patients who had not visited our institution, including other departments, in the last 2 years were considered lost to follow-up and excluded. Therefore, a total of 165 patients were analyzed. The median exposure time to implants was 5.5 years. As for the type of reconstructive surgery, a latissimus dorsi musculocutaneous flap with an implant was the most common procedure, accounting for $57.0 \%$ (Table 1). Interestingly, only $6.7 \%$ of the patients had regularly scheduled outpatient follow-up visits before the occurrence of the first BIA-ALCL case report in Korea and text message transmission. The rate of outpatient visits to our department temporarily increased by about five times to $33.9 \%$ after the first BIA-ALCL case report in Korea and text message transmission.

Fifty-six patients (33.9\%) visited our outpatient office within the year after the first BIA-ALCL case report (Fig. 3). More than half of them visited the outpatient office during the initial 4 months. In particular, the number of outpatient visits was the highest in the 2 months after the message was sent and the number of visitors decreased thereafter. None of the patients were suspected of having BIA-ALCL on physical examination and imaging studies.

Table 1. Types of surgery underwent by patients who received implant-based breast reconstruction

\begin{tabular}{|c|c|c|}
\hline Purpose of surgery & Subtype & $\begin{array}{c}\text { No. of } \\
\text { patients }(\%)\end{array}$ \\
\hline \multirow{6}{*}{$\begin{array}{l}\text { Reconstructive } \\
\text { surgery }\end{array}$} & DTI & 25 (15.2) \\
\hline & DTI/contralateral augmentation & $13(7.9)$ \\
\hline & LD+implant & $94(57.0)$ \\
\hline & LD+implant/contralateral augmentation & $28(17.0)$ \\
\hline & Both DTI & $5(3.0)$ \\
\hline & Total & 165 \\
\hline
\end{tabular}

DTI, direct to implant; LD, latissimus dorsi musculocutaneous flap. 
Among the patients who underwent reoperation, there were no cases wherein BIA-ALCL was suspected based on the intraoperative findings. Total capsulectomy was done for severe capsular contracture in only one patient, while in six patients, partial capsulectomy was done for double capsule formation. As a result, no pathological findings suspicious of BIA-ALCL were observed in the resected capsules. Complications other than BIA-ALCL in 12 patients were incidentally found through additional outpatient office visits and treated by reoperation. In 11 of these patients, the implant was exchanged with a smooth implant according to the recommendation, while one patient underwent only removal of the textured implant. The textured implant was replaced with a smooth implant without changing the pocket. Only three of the outpatient visitors did not follow the recommendations due to concerns about BIAALCL and underwent prophylactic surgery. All of them underwent removal of the implant, in discordance with the recommendations

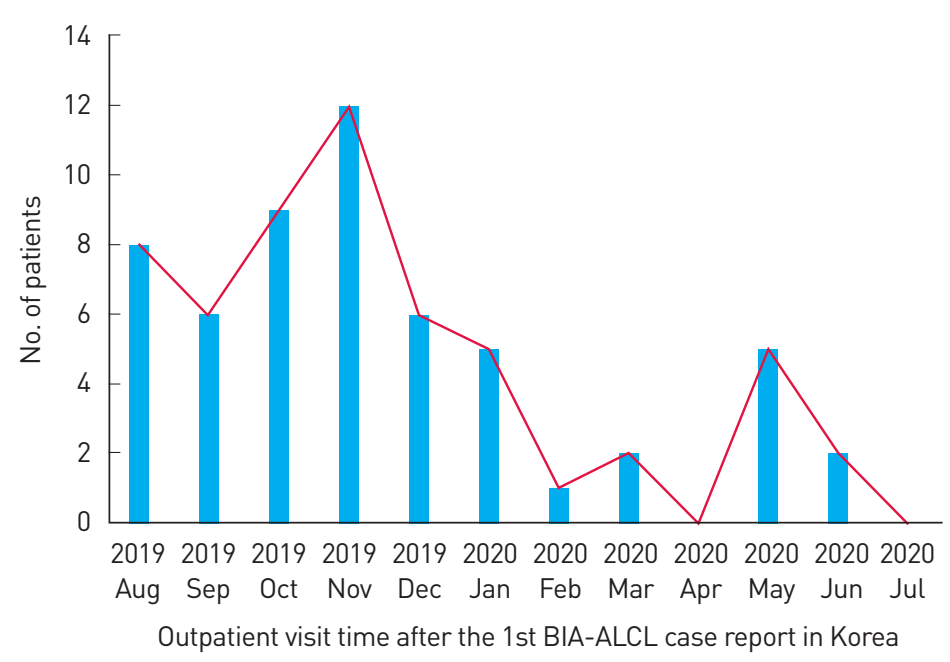

Fig. 3. The number of patients who made outpatient visits after the first breast implant-associated anaplastic large cell lymphoma (BIAALCL) case report in Korea.
(Table 2). The proportion of patients who underwent implant exchange with a smooth implant, according to recommendations, was $76.7 \%$ in patients who underwent reoperation after the first BIA-ALCL case report. In a comparison of trends before and after the first BIA-ALCL case, no statistically significant difference was noted in the ratio of exchange and removal of textured implants $(\mathrm{P}=0.700)$.

\section{DISCUSSION}

Since the first BIA-ALCL case was reported in 1997, the U.S. FDA stated that 733 medical device reports of BIA-ALCL cases had been identified worldwide by 2020 [11,12]. In Korea, reconstruction and cosmetic surgery using breast implants is actively conducted, and in August 2019, the first BIA-ALCL case was reported in a patient with textured implants (Allergan, Inc.) [10]. After the first case report, the second and third patients in Korea were reported by October 2020 [13]. As this situation was provocatively reported through some media outlets, patients complained of severe psychological anxiety, which made them consider reoperation, and some hospitals rose to meet this demand. The inconsistent treatment policies of medical personnel also increased confusion. To solve this problem, the KSPRS and KMFDS officially presented recommendations based on previous research results. Algorithms for diagnosing and treating BIA-ALCL were recommended in the National Comprehensive Cancer Network guidelines [1]. Patients with symptoms such as swelling, pain, or rash were recommended to undergo imaging tests. In previous studies, the incidence of BIA-ALCL was reported to be low, although it could increase with longer exposure time to implants $[3,5,6]$. If diagnosed early, most BIA-ALCL patients show an excellent prognosis with complete surgical excision including capsulectomy $[1,14]$. Therefore, the prophylactic removal of implants is not generally recommended for asymptomatic patients, and it is assumed that patients receive regular follow-up with specialists.

Table 2. Indications for reoperation before and after the first BIA-ALCL case report

\begin{tabular}{|c|c|c|c|c|c|}
\hline Indication for reoperation & \multicolumn{2}{|c|}{ Before the first BIA-ALCL $(n=20)$} & \multicolumn{2}{|c|}{ After the first BIA-ALCL ( $n=15$ ) } & P-value \\
\hline Implant rupture & $7(35.0)$ & $1(5.0)$ & 8 (53.3) & $1(6.7)$ & - \\
\hline Capsular contracture & $6(30.0)$ & 0 & $1(6.7)$ & 0 & - \\
\hline Infection/inflammation & $1(5.0)$ & $3(15.0)$ & $1(6.7)$ & 0 & - \\
\hline Recurrence of breast cancer & $1(5.0)$ & 0 & $1(6.7)$ & 0 & - \\
\hline Prophylactic surgery & 0 & 0 & 0 & $3(20.0)$ & - \\
\hline Total & $16(80.0)$ & $4(20.0)$ & 11 (73.3) & $4(26.7)$ & 0.700 \\
\hline
\end{tabular}

BIA-ALCL, breast implant-associated anaplastic large cell lymphoma.

The Fisher exact test was used to test the significance of differences in the rate of implant removal between before and after the Allergan issues. 
Most patients who visited the outpatient office listened to the explanations and asked questions, demonstrating high compliance intent. There were no cases of suspected BIA-ALCL among the outpatients in the present study, and no cases of suspected or confirmed BIA-ALCL by biopsy after reoperation. Asymptomatic patients who did not follow the recommendations and underwent a prophylactic reoperation wanted to remove the implants, even though replacement with a smooth implant was recommended. However, these patients represented only 5.4\% of our outpatient visitors after the first BIA-ALCL case. This was a surprising result as we thought that reconstructive patients would be particularly sensitive to BIAALCL concerns because of their past treatment for cancer. The fact that the outpatients visited within 1 year of receiving the text message may be an indicator that this patient group was already highly compliant. However, even considering this, the rate of preventive reoperations was considered to be quite low. In the U.S., $10 \%$ of survey respondents with breast implants said that they were very worried about BIA-ALCL and 35\% said they strongly considered implant removal despite the information provided in the current U.S. FDA guidelines [15]. This may be an indicator that direct communication is very important. Steve et al. [16] suggested that patients' psychological concerns about BIA-ALCL could be arranged in five themes (listening, informing, acknowledging, clarifying, and moving forward) based on the trust theory. They reported that patients wanted plastic surgeons to provide information regarding adverse outcomes, to listen sincerely without disregarding their concerns, and to have a clear plan according to the individual situations. In addition, they said that there were apparent gaps between the opinions of patients and surgeons, which could be overcome through trust in plastic surgeons. Through this approach to patients, unnecessary surgery can be reduced, and complications can be managed.

No seroma or mass-like lesion in the capsules suspicious of BIAALCL was observed in patients who underwent reoperations at our institution. However, double capsules were often observed, which is thought to be consistent with previous studies where double capsules were found in a higher proportion in Biocell textured implants than in smooth implants $[17,18]$, presumably due to the effect of the rough texture of the implant [17]. Biopsy was also performed while removing these parts, but no specific findings were observed. Treatment for BIA-ALCL includes removal of the implant with the surrounding capsule and mass-like lesion $[1,14]$. However, there is no known benefit of prophylactic capsulectomy in asymptomatic patients. Total capsulectomy was not performed in the patients in this study who underwent reoperations, including those for prophylactic purposes, except for one patient with grade 4 capsular contracture. Considering the risks and benefits of surgery and anesthesia, a previous study reported that capsulectomy was not essential when removing implants or replacing smooth implants in asymptomatic patients [19].
One unanticipated finding was that the periodic follow-up rate in our department was $6.7 \%$ before the first domestic BIA-ALCL case report even though our institution recommended periodic follow-up with the general surgery department. Our department recommends follow-up observations at 1 week, 1 month, 6 months, and 12 months after surgery, and outpatient visits every year thereafter. A similarly low proportion was identified among patients who visited other departments of our hospital over the past 2 years, which means that most patients were unaware of the need to visit our outpatient office periodically and we also overlooked the need for regular follow-up. After the first case report of BIA-ALCL and text message transmission, this rate increased by five times, but still only included one-third of all patients. Lee et al. [15] showed a low level of public awareness of BIA-ALCL in the U.S. despite numerous media reports. They reported that only $13.6 \%$ of the women in the U.S. (including $51.7 \%$ of the respondents with implants) had heard of BIA-ALCL.

The number of outpatients increased after text messages were sent, especially in the first 4 months (Fig. 3). However, a limitation is that it is difficult to know the exact effect of the Allergan issues and message transmission. In addition, it is possible that there may have been almost no additional outpatient office visitors thereafter, as the number of outpatient visits subsequently decreased. Nonetheless, for periods beyond 1 year, patients' responses to our recommendation of once-a-year outpatient observations should be checked.

The incidence of BIA-ALCL in patients with textured implants was 1.79 per 1,000 patients and increased over time, especially in patients with over 10 years of exposure to implants [6]. In this study, the median exposure time to implants was 5.5 years, so BIA-ALCL may not have developed yet. Therefore, follow-up observations in the future are considered to be particularly important. In addition to BIA-ALCL, several other diseases-known as breast implant illnesses-can be caused by breast implants [20]. After the report of the first case of BIA-ALCL, 12 patients in 1 year received reoperations due to complications found incidentally at outpatient visits. Most of these patients agreed to have the implant replaced with a smooth-type implant. Out of 240 patients who had undergone breast surgery with Biocell textured implants, 20 had undergone reoperations over the 5 years before the report of the first case of BIA-ALCL. Thus, it is difficult to accurately compare the two groups of patients, but this study identified many patients in need of surgery for breast implant illnesses other than BIA-ALCL through additional outpatient follow-up visits. Among the patients who underwent reoperation after the Allergan issues, $76.7 \%$ received exchange with other implants, which was not substantially different from the rate before the Allergan issues. Even before the Allergan issues, we recommended implant exchange rather than removal for reconstructed breasts, and this recommendation continued to be made after the Allergan issues. Some patients had negative thou- 
ghts about all breast implants, including textured implants, and wanted to remove them, but most of them were persuaded to undergo replacement through counseling. Therefore, these results may show that the impact of the Allergen issues could be moderated to some extent through our recommendations.

In our institution, phone text messages were delivered to patients with Biocell textured implants to encourage visits to the outpatient office. Roberts et al. [21] reported a prospective study of patients with breast implants and demonstrated that informing patients of the risks of BIA-ALCL was an important endeavor. This was similar to our study in that patients' responses were observed at outpatient visits and the rate of reoperations after delivering the information to the patients was investigated. However, the patients in our study were influenced by the first BIA-ALCL case report in Korea and were limited to patients who had undergone reconstruction with textured breast implants (Allergan, Inc.). Most of the patients with implants in our institution had undergone breast reconstruction after mastectomy. Unlike cosmetic surgery patients, most of the patients who had received implants for reconstruction purposes were being regularly followed up at the general surgery department at least once a year, making it relatively easy to contact them and convey the recommendations. Lee et al. [15] showed that information was mainly obtained through health professionals, blogs, and media, and the proportion of women receiving information from different sources, obtained through a survey asking American women about BIA-ALCL, was reported to be similar. They also reported that there were no differences in patients' concerns according to the type of information source. Therefore, for patients who do not visit outpatient offices regularly, health professional blogs or popular social media may be effective for continued information transfer.

It is difficult for individual doctors and hospitals to respond and take responsibility for all of these medical device-related issues. Nationally, there is a need for a system that can consistently track patients who use implants and manage their safety in the future. In 2012, the American Society of Plastic Surgeons, the Plastic Surgery Foundation, and the U.S. FDA signed a cooperative agreement to develop a patient registry entitled the "Patient Registry and Outcomes for Breast Implants and Anaplastic Large Cell Lymphoma Etiology and Epidemiology" (PROFILE) [22]. The specific purposes of the PROFILE Registry are to determine the recurrence rate and overall survival rate by collecting the demographic, clini$\mathrm{cal}$, and pathological features of patients diagnosed with BIA-ALCL. In addition to the U.S., several countries have established similar breast implant registries, and national plastic surgery societies have gathered to form an international collaboration [23]. After the first BIA-ALCL case in Korea, a pilot project was undertaken through collaboration between the KSPRS and KMFDS. Therefore, it is necessary to plan and implement a patient registration and long-term tracking management system by reviewing similar cases according to the international situation and applying examples from other countries to Korea.

This study had several limitations. It was a retrospective study that relied on chart reviews at a single institution, and it was difficult to confirm accurate information on the patients who had not visited the outpatient clinics. In addition, this study confirmed only the results for 1 year after the first BIA-ALCL case, and further research is needed to confirm whether periodic follow-up is performed in the future.

This study is significant in that it is the first study on the responses of patients with breast reconstructions with a Biocell textured implant after the first BIA-ALCL case report in Korea. Most patients who visited our outpatient office showed high compliance with our recommendations, but there was a lower rate of periodic outpatient visits than we expected. Therefore, it is necessary to improve delivery and management methods to inform patients appropriately and explain the need for periodic observations of progress. Accordingly, multi-center and long-term studies are needed to confirm follow-up observation and management. As patients' concerns regarding a series of implant-related issues are spreading, a responsible attitude on our part is required to provide accurate information, ensure regular follow-up observations of patients with breast implants, and establish a monitoring system for them.

\section{NOTES}

\section{Conflict of interest}

No potential conflict of interest relevant to this article was reported.

\section{Ethical approval}

The study was approved by the Institutional Review Board of Soonchunhyang University Hospital (IRB No. 2021-01-011) and performed in accordance with the principles of the Declaration of Helsinki. Written informed consent was obtained.

\section{ORCID}

Ji Min Kim

Woo Jin Song

Hyun Gyo Jeong

Sang Gue Kang

https://orcid.org/0000-0001-5782-8958 https://orcid.org/0000-0002-8957-3950 https://orcid.org/0000-0002-6378-6093 https://orcid.org/0000-0001-8055-6591

\section{REFERENCES}

1. Clemens MW, Jacobsen ED, Horwitz SM. 2019 NCCN Consensus Guidelines on the diagnosis and treatment of breast implant-associated anaplastic large cell lymphoma (BIA-ALCL). Aesthet Surg J 2019;39 (Suppl_1):S3-13.

2. Brody GS, Deapen D, Taylor CR, et al. Anaplastic large cell lymphoma occurring in women with breast implants: analysis of 173 cases. Plast Reconstr Surg 2015;135:695-705. 
3. Collett DJ, Rakhorst H, Lennox P, et al. Current risk estimate of breast implant-associated anaplastic large cell lymphoma in textured breast implants. Plast Reconstr Surg 2019;143(3S):30S-40S.

4. Clemens MW, Brody GS, Mahabir RC, et al. How to diagnose and treat breast implant-associated anaplastic large cell lymphoma. Plast Reconstr Surg 2018;141:586e-599e.

5. Doren EL, Miranda RN, Selber JC, et al. U.S. epidemiology of breast implant-associated anaplastic large cell lymphoma. Plast Reconstr Surg 2017;139:1042-50.

6. Nelson JA, Dabic S, Mehrara BJ, et al. Breast implant-associated anaplastic large cell lymphoma incidence: determining an accurate risk. Ann Surg 2020;272:403-9.

7. U. S. Food \& Drug administration (FDA). FDA takes action to protect patients from risk of certain textured breast implants; requests Allergan voluntarily recall certain breast implants and tissue expanders from market [Internet]. Silver Spring, MD: Food and Drug Administration; c2019 [cited 2021 Jan 27]. Available from: https://www.fda.gov/newsevents/press-announcements/fda-takes-action-protect-patients-riskcertain-textured-breast-implants-requests-allergan.

8. Torres E. Allergan issues global recall of breast implants linked to rare cancer [Internet]. New York, NY: ABC News; c2019 [cited 2021 Jan 27]. Available from: https://abcnews.go.com/Health/allergan-issuesglobal-recall-breast-implants-linked-rare/story?id=64544381.

9. Lee CJ. More than 110,000 number of Allergan textured implants distributed in Korea [Internet]. Seoul: Medicaltimes; c2019 [cited 2021 Jan 21]. Available from: https://medicaltimes.com/Users/News/NewsView.html?ID=1128366.

10. Kim IK, Hong KY, Lee CK, et al. Analysis of the molecular signature of breast implant-associated anaplastic large cell lymphoma in an Asian patient. Aesthet Surg J 2021;41:NP214-22.

11. Keech JA Jr, Creech BJ. Anaplastic T-cell lymphoma in proximity to a saline-filled breast implant. Plast Reconstr Surg 1997;100:554-5.

12. Clemens M. BIA-ALCL Resources: by the numbers, and what they mean [Internet]. Arlington Heights, IL: American Society of Plastic Surgeons; c2020 [cited 2021 Jan 29]. Available from: https://www.plas- ticsurgery.org/for-medical-professionals/health-policy/bia-alcl-physician-resources/by-the-numbers.

13. Gye SH, Kim JD. Unfinished Allergan breast implant fear... the third rare cancer in Korea [Internet]. Seoul: Yonhapnews; c2020 [cited 2021 Jan 29]. Available from: https://www.yna.co.kr/view/AKR202010040 30000017.

14. Campanale A, Spagnoli A, Lispi L, et al. The crucial role of surgical treatment in BIA-ALCL prognosis in early- and advanced-stage patients. Plast Reconstr Surg 2020;146:530e-538e.

15. Lee E, Khavanin N, He W, et al. Public perceptions on breast implantassociated anaplastic large cell lymphoma. Plast Reconstr Surg 2020; 146:30-7.

16. Steve AK, Temple-Oberle C, Yeung JK, et al. "You helped create this, help me now": a qualitative analysis of patients' concerns about breast implants and a proposed strategy for moving forward. Plast Reconstr Surg 2021;147:16e-24e.

17. Giot JP, Paek LS, Nizard N, et al. The double capsules in macro-textured breast implants. Biomaterials 2015;67:65-72.

18. Hall-Findlay EJ. Breast implant complication review: double capsules and late seromas. Plast Reconstr Surg 2011;127:56-66.

19. Swanson E. Evaluating the necessity of capsulectomy in cases of textured breast implant replacement. Ann Plast Surg 2020;85:691-8.

20. Magnusson MR, Cooter RD, Rakhorst $H$, et al. Breast implant illness: a way forward. Plast Reconstr Surg 2019;143(3S):74S-81S.

21. Roberts JM, Carr LW, Jones A, et al. A prospective approach to inform and treat 1340 patients at risk for BIA-ALCL. Plast Reconstr Surg 2019; 144:46-54.

22. McCarthy CM, Loyo-Berrios N, Qureshi AA, et al. Patient Registry and Outcomes for Breast Implants and Anaplastic Large Cell Lymphoma Etiology and Epidemiology (PROFILE): initial report of findings, 2012-2018. Plast Reconstr Surg 2019;143(3S):65S-73S.

23. Spronk PER, Begum H, Vishwanath S, et al. Toward international harmonization of breast implant registries: International Collaboration of Breast Registry Activities global common data set. Plast Reconstr Surg 2020;146:255-67. 